\title{
Star Formation in the LMC: Comparative CCD Observations of Young Stellar Populations in two Giant Molecular Clouds
}

\author{
Jan Ruppert ${ }^{1}$ and Hans Zinnecker ${ }^{2}$ \\ ${ }^{1}$ European Southern Observatory, \\ Alonso de Cordova 3107, Vitacura, Santiago, Chile \\ email: jruppert@eso.org \\ ${ }^{2}$ Astrophysikalisches Institut Potsdam, \\ An der Sternwarte 16, D-14482 Potsdam, Germany \\ email: hzinnecker@aip.de
}

\begin{abstract}
This work deals with a CCD imaging study at optical and near-infrared wavelength of two giant molecular clouds (plus a control field) in the southern region of the Large Magellanic Cloud, one of which shows multiple signs of star formation, whereas the other does not. The observational data from VLT FORS2 $(R$-band) and NTT SOFI ( $K s$-band) have been analyzed to derive luminosity functions and color-magnitude diagrams. The young stellar content of these two giant molecular clouds is compared and confirmed to be different, in the sense that the apparently "starless" cloud has so far formed only low-luminosity, low-mass stars (fainter than $m_{K s}=16.5 \mathrm{mag}$, not seen by 2MASS), while the other cloud has formed both faint low-mass and luminous high-mass stars. The surface density excess of low-luminosity stars $(\sim 2$ per square arcmin) in the "starless" cloud with respect to the control field is about $20 \%$ whereas the excess is about a factor of 3 in the known star-forming cloud. The difference may be explained theoretically by the gravo-turbulent evolution of giant molecular clouds, one being younger and less centrally concentrated than the other.
\end{abstract}

Keywords. stars: formation, luminosity function, ISM: clouds, evolution, HII regions, galaxies: stellar content, infrared: stars

\section{Introduction}

This contribution presents a brief summary of our results comparing observations of the young stellar content of two distinct giant molecular clouds (GMCs) in the LMC. One of these GMCs is an actively star-forming GMC whereas the other GMC was classified as "starless" by Fukui et al. (2001) and Mizuno et al. (2001), due to a lack of e.g. HiI regions. The full article describing our observations and the data reduction process as well as the results and their discussion can be found in Ruppert \& Zinnecker (2009).

\section{Overview of Results}

Our observations in the optical and the near-infrared have shown that the evolutionary sequence for low-metallicity GMCs by Fukui (2005) do not give us the full picture as it did not consider an underlying low-mass star-forming population. The overall stellar surface density found in the "starless" GMC is statistically approximately $20 \%$ above the mean stellar surface density found in the control field where in comparison the stellar surface density in the star-forming GMC was about three times higher than in the control field. The infrared K-band luminosity functions in the "star-less" and star-forming GMC are shown in Fig. 1 (top = FORS2 upper chip matching SOFI data, bottom = lower chip). 


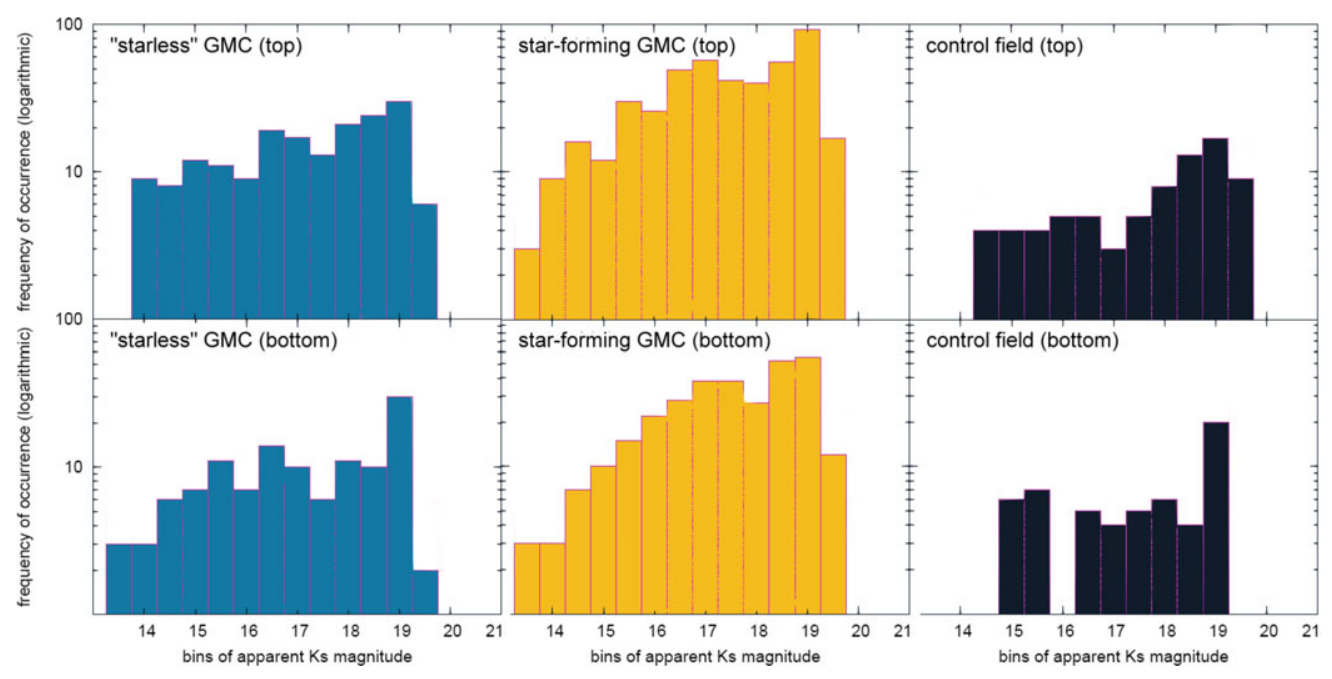

Figure 1. Stellar luminosity distribution

The log-plots for all three observed fields account only for SOFI objects with matched FORS2 positions, but with giant branch and objects below the sensitivity limit in the $K s$-band removed.

\section{Implications on Low-Metallicity Star Formation}

The main reason why some GMCs in the LMC have been described as "starless" by Fukui et al. (2001) is the non-detection of His regions in them (no $\mathrm{H} \alpha$ or reflection nebulae). However, this does not exclude a young low-mass stellar population with apparent brightnesses fainter than $m_{K s} \sim 16.5 \mathrm{mag}\left(\mathrm{M} \star<10 \mathrm{M}_{\odot}\right)$, i.e. objects very common in known star-forming GMCs which do not create visible HiI regions. Our observations now reveal that low-level, low-mass star formation has indeed already occurred in one of the "starless" GMCs, and that low-mass star formation increases substantially (factor $\sim 3$ ) by the time massive stars and HiI regions appear, as seen in another ("star-forming") GMC. Thus Fukui's conclusion is due to an observational selection effect, and his simple evolutionary sequence for low-metallicity GMCs from "starless" to "star-forming" is missing an underlying low-mass star-forming population. Therefore, it seems, contrary to Fukui's conclusion, that the low-metallicity GMCs in the LMC evolve in quite a similar way as solar-metallicity GMCs in the Milky Way. Indeed, Fukui's "starless" GMCs in the LMC, see Blitz et al. (2007), resemble the case of Maddalena's GMC in the Milky Way which was once believed to be starless, but recently low-level low-mass star formation has been discovered in it (Megeath et al. 2009). Gravo-turbulent evolution of GMCs, largely independent of metallicity, may explain the above results (MacLow \& Klessen 2004).

\section{References}

Blitz, L. et al. 2007, in: Reipurth, B., Jewitt, D., Keil, K. (eds.), Protostars and Planets V (Tucson: University of Arizona Press), 951, 81-96

Fukui, Y. et al. 2001, PASJ, 53, L41-L44

Fukui, Y. 2005, in: Cesaroni, R., Felli, M., Churchwell, E., Walmsley, M. (eds.), IAU Symposium Proceedings (Cambridge: Cambridge University Press), 227, 328-336

Mizuno, N. et al. 2001 PASJ, 53, 971-984

MacLow, M.-M. \& Klessen, R. S. 2004, RvMP, 76, 125-194

Megeath, S. T. et al. 2009, AJ, 137, 4072-4082

Ruppert, J. \& Zinnecker, H. 2009, AN, 330, 773-783 